\title{
Efficacy measurement of ketorolac in reducing the severity of headache
}

\author{
Alireza Baratloo', Marzieh Amiri², Mohammad Mehdi Forouzanfar', Sadegh Hasani³, Samar Fouda4, \\ Ahmed Negida ${ }^{*}$
}

'Department of Emergency Medicine, Shohadaye Tajrish Hospital, Shahid Beheshti University of Medical Sciences, Tehran, Iran ${ }^{2}$ Department of Emergency Medicine, Shahid Beheshti Hospital, Guilan University of Medical Sciences, Anzali, Iran ${ }^{3}$ Faculty of Medicine, Shahid Beheshti University of Medical Sciences, Tehran, Iran ${ }^{4}$ Faculty of medicine, Zagazig University, Zagazig, Egypt

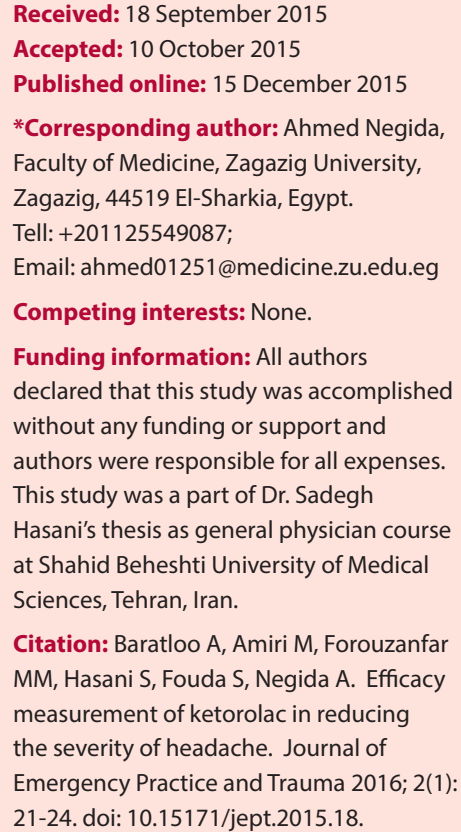

\begin{abstract}
Objective: One of nonsteroidal anti-inflammatory drugs (NSAIDs) named as ketorolac is frequently used to relieve acute pain. Current study was conducted with the aim of ketorolac efficacy measurement as a pain killer agent for controlling the primary headache in emergency departments.

Methods: In this study, we enrolled 50 patients with primary headache who received $60 \mathrm{mg}$ ketorolac intravenously as a slow infusion in about 10 minutes. Pain scores were evaluated with visual analog scale (VAS) on arrival and also 1 hour and 2 hours after ketorolac infusion. Statistical analysis was performed on collected data by using Wilcoxon and Mann-Whitney tests to assess the differences in VAS pain scores.

Results: Decreasing the VAS more than 3 points from the arrival until 1 hour $(P<0.001)$, and more than 5 points from the arrival until 2 hours after ketorolac administration $(P<0.001)$ were seen. Those with history of analgesic use before admission in emergency department in comparison with the others did not accompany with more decline in pain score after 1 hour $(P=0.34)$ or 2 hours $(P=0.92)$.

Conclusion: It seems that ketorolac is assured, safe and well tolerated agent for pain control in patients presented with primary headache to the emergency departments. Based on the results achieved in this study, ketorolac illustrates its perceptible effects within 1 hour after administration that even more prominent after 2 hours. Keywords: Ketorolac, Headache, Pain management, Emergency medicine
\end{abstract}

\section{Introduction}

Headache is an omniscient illness with a lifetime prevalence rate reaching 90\% (1). There are several types of headaches, so that 150 diagnostic headache categories have been defined. Most patients arriving in the emergency department (ED), suffering from severe headache require a rapid and an effective release from their symptoms $(2,3)$. There are frequent guidelines for management of acute headaches that generally recommended several different agents, such as dihydroergotamine, triptans, phenothiazines, opioids or nonsteroidal anti-inflammatory drugs (NSAIDs) (4-6). Ketorolac is one of NSAIDs which has been tested several times in randomized clinical trials and recommended as an effective agent for patients with pain (7-11). However the search is still on. Ketorolac accepted as an alternative to non-steroidal analgesics and also opioid in controlling moderate to severe pain (12). About 2 decades before, it was thought that although ketorolac has a considerable analgesic effect, but has restriction for ap- plication as a fast pain abortive in the ED (13). Some other believed that this drug could be considered as a useful adjuvant, or alternative for opioids in subjects with moderate to severe pain $(14,15)$. Thinking that different types of headaches involve the same inflammatory component in their pathophysiology, ketorolac is expected to be effective. This agent is an efficient non-narcotic pain killer with intermediate anti-inflammatory effect. Therefore consider as a preferable option for pain management of different type of primary headache cases admitted to the ED (16-19). Current study was conducted with the aim of ketorolac efficacy measurement as a non-narcotic antiinflammatory pain killer agent for controlling the primary headache in of patients presented to the ED with all types of primary headaches.

\section{Methods}

Patients with any kind of primary headache admitted to the ED of Shohadaye Tajrish hospital in Tehran, Iran were 
participated in this study.

All patients in 18-60 years old age range with complaint of moderate to severe headache (pain score $>5$ ) that indicated for intravenous treatment were included.

Patients with positive history for any of the following items were excluded: breast feeding, pregnancy, active peptic ulcer disease, coagulopathy, inflammatory bowel disease, renal failure or hepatic failure.

The pain scores were recorded on arrival based on visual analog scale (VAS) and those with the score more than 5 received $60 \mathrm{mg}$ ketorolac intravenously as a slow infusion in about 10 minutes. All were considered for any possible side effects including irritability, itching, pain on injection, hypertension, tachycardia, nausea and/or vomiting. If any happened then process had to be stop. One and 2 hours after injection of medication to the patients, they were asked about their pain scores again. Decreasing more than 3 scores in pain scale was considered as proper response.

SPSS version 22 was used for performing the statistical analysis. For describing continuous variables mean, median, interquartile range, and standard deviation were used. For analyzing the differences in registered pain scores, Mann-Whitney U and Wilcoxon tests were used. For describing categorical variables, frequency and percentage were used. $P \leq 0.05$ was considered as meaningful level.

\section{Results}

Fifty patients with the mean age of $30.14 \pm 11.4$ years old were involved. From the participants, 22 patients (44\%) were male and 28 patients (56\%) were female. It should be mentioned that $28(56 \%)$ patients did not use any kind of analgesic before arrival to the ED. From other 22 patients (44\%) who had already taken other medication, almost all of them had used acetaminophen or different type of NSAIDs. The mean \pm standard deviation period from taking other medication to ketorolac administration was $2.77 \pm 1.9$ hours. The VAS median reduced significantly from $8.0 \pm 2.0$ to $5.0 \pm 4.0$ after 1 hour and to $3.0 \pm 2.0$ after 2 hours (Figure 1).

Significant VAS drop (>3 points, $>35 \%$ ) was reported from baseline until 1 hour after ketorolac administration (Wilcoxon test $\mathrm{Z}=-6.1 ; P<0.001$ ). One hour later, more decline ( $>6$ points, $>60 \%$ ) was seen (Wilcoxon test $\mathrm{Z}=-6.1 ; P<0.001)$. There was also a statistical significant difference between VAS at 1 hour and 2 hours after drug administration (Wilcoxon test $\mathrm{Z}=-4.2 ; P<0.001$ ).

To assess the possible effect of medication use before arrival to the $\mathrm{ED}$, enrolled patients were divided into 2 groups according to taking previous medication or not. Statistical analysis did not reveal significant difference between the groups. The $\mathrm{P}$ values related to comparison of VAS between two groups at baseline, 1 hour and 2 hours after ketorolac administration were $0.51,0.62$, and 0.69 , respectively. There was also no significant statistical difference between the groups in terms of VAS reducing 1

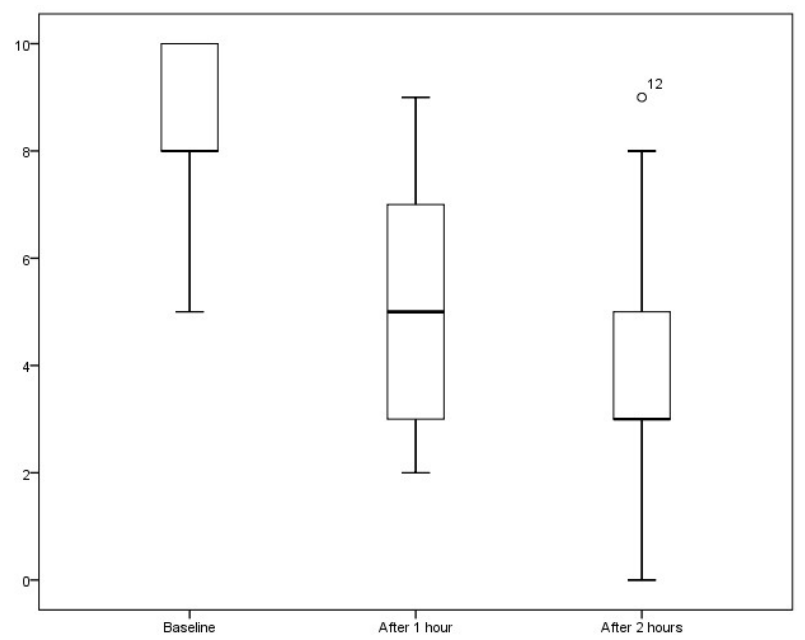

Figure 1. Minimum, maximum, median and interquartile range of visual analogue scale at baseline, 1 hour and 2 hours after ketorolac intravenous infusion.

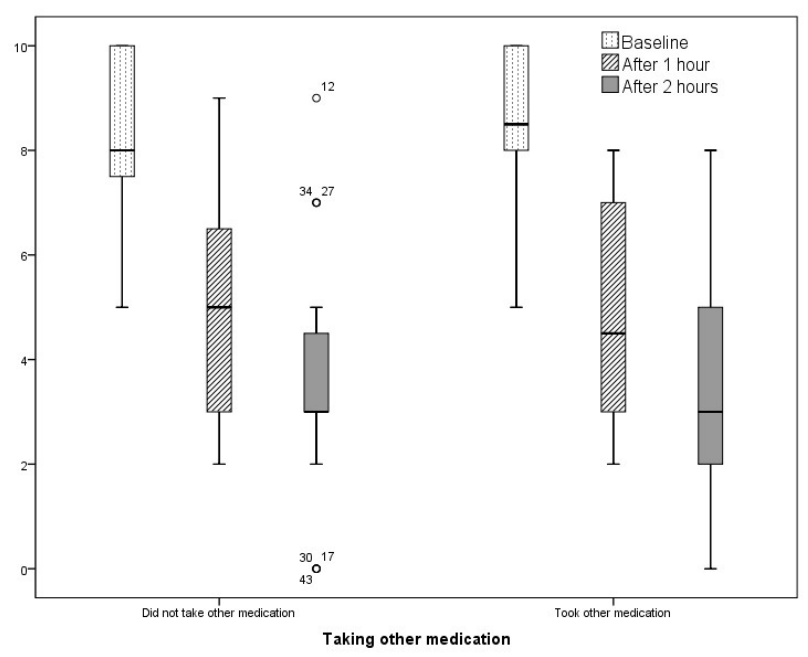

Figure 2. Minimum, maximum, median and interquartile range of visual analogue scale at baseline, one hour and two hours after ketorolac intravenous infusion for patients who took analgesic before arrival and those who did not.

hour $(P=0.34)$ and 2 hours $(P=0.92)$ after ketorolac administration (Figure 2).

\section{Discussion}

Based on findings of the current study, significant decreasing of pain score was seen from baseline to 1 hour and 2 hours after ketorolac administration. This improvement was obtained in almost all cases except two patients whose VAS did not improve well even after 2 hours. The intravenous ketorolac accompanied with significant success rate so that lead to more than 3 points decline in VAS after one hour and more than 5 points after 2 hours.

All patients were observed for about 6 hours after drug administration for possible short time side effects and also replace of headache. Fortunately, there was no case of such complications, which can confirm the safety and effec- 
tiveness of intravenous ketorolac in the enrolled patients. According the rigorous intended inclusion and exclusion criteria for selecting patients in this study, results should be interpreted with caution.

Parenteral ketorolac is frequently used for abortive treatment of moderate to severe headache. As a NSAID, ketorolac mechanism of action is inhibition of prostaglandin synthesis by non-selective inhibition of cyclooxygenase enzyme.

Ketorolac were used in several studies, so supporting and opposing comments has been proposed. In a pilot study, $60 \mathrm{mg}$ intramuscular ketorolac was administered to twelve patients with headache crisis. All patients showed statistically significant improvement on their McGill pain questionnaire and authors recommended this drug as a possible useful agent in the treatment of such patients (20).

Intravenous ketorolac was compared with nasal sumatriptan in a prospective double-blind study that performed on patients with complaint of migraine headache. Authors reported that although both drugs significantly reduced the pain, but intravenous ketorolac was more effective than nasal sumatriptan in this regard (21).

In a controlled trial, intramuscular ketorolac was compared with meperidine plus promethazine, and also normal saline as a placebo in tension headache. The authors reported that ketorolac was superior to placebo at 0.5 and 1 hour and superior to meperidine at 2 hours (22). Interestingly, these authors had conducted the same study on acute headache crises 2 years earlier and had reported that using all three options could lead to significant pain control, but the amount did not differ among them (23).

In a non-interventional non-randomized survey on pain management, intramuscular ketorolac and oral ibuprofen were compared. The authors of the mentioned study concluded that both option produced similar pain relief in dealing with acute pain in ED. They believed that ketorolac did not have certain superiority than ibuprofen for this aim (24).

In another study which performed on patients presenting with migraine headache, it was shown that one dose injection of ketorolac can lead to significant decrease in headache symptoms after 1 hour in most participants (25).

In a randomized trial for comparing the effectiveness of intravenous ketorolac, metoclopramide, and valproate sodium for management of acute migraine headache, it was concluded that ketorolac was superior to valproate sodium but less efficacious than metoclopramide (26).

Intravenous ketorolac was also compared with intravenous diphenhydramine plus metoclopramide for treatment of some primary headaches. The study concluded that for adults who presented to an ED with non-migraine, non-cluster primary headaches, intravenous diphenhydramine plus metoclopramide have better efficacy than intravenous ketorolac (27).

Using a standard measuring tool like VAS and the precise selection of patients can be considered as the strengths in this study. On the other hand, short time follow up period is one of the limitations. Considering a control group, escalating doses, larger population, and describing nonresponders patients to ketorolac are recommended for future research.

\section{Conclusion}

It seems that ketorolac is assured, safe and well tolerated agent for pain control in patients presented with primary headache to the EDs. Based on the results achieved in this study, ketorolac illustrates its perceptible effects within 1 hour after administration that even more prominent after 2 hours.

\section{Acknowledgements}

We would like to express our special thanks to the ED staff of Shohadaye Tajrish hospital, Tehran, Iran.

\section{Ethical issues}

This study was done with respect of Declaration of Helsinki - Ethical Principles for Medical Research Involving Human Subjects. All eligible patients were enrolled only after signing the informed consent. Study protocol was assessed and approved by the ethical committee of Shahid Beheshti University of Medical Sciences, Tehran, Iran. This study is a fundamental part of a clinical trial registered at Iranian Registry of Clinical Trials (code: IRCT2013120315640N1).

\section{Authors' contributions}

All authors passed four criteria for authorship contribution based on recommendations of the International Committee of Medical Journal Editors. This study was a part of Dr. Sadegh Hasani's thesis as general physician course at Shahid Beheshti University of Medical Sciences, Tehran, Iran.

\section{References}

1. Frishberg BM. Neuroimaging in presumed primary headache disorders. Semin Neurol 1997; 17(4): 37382.

2. Davis CP, Torre PR, Williams C, Gray C, Barrett K, Krucke G, et al. Ketorolac versus meperidine-pluspromethazine treatment of migraine headache: evaluations by patients. Am J Emerg Med 1995; 13(2): 146-50.

3. Kasmaei HD, Baratloo A, Soleymani M. A 33-yearold woman with severe postpartum headache. Emergency 2013; 1(1): 27-9.

4. Silberstein SD. Practice parameter: evidence-based guidelines for migraine headache (an evidence-based review) report of the Quality Standards Subcommittee of the American Academy of Neurology. Neurology 2000; 55(6): 754-62. doi: 10.1212/WNL.55.6.754.

5. Snow V, Weiss K, Wall EM, Mottur-Pilson C. Pharmacologic management of acute attacks of 
migraine and prevention of migraine headache. Ann Intern Med 2002; 137(10): 840-9. doi: 10.7326/00034819-137-10-200211190-00014.

6. Baratloo A, Negida A, El Ashal G, Behnaz N. Intravenous caffeine for the treatment of acute migraine: a pilot study. J Caffeine Res 2015; 5(3): 1259. doi: 10.1089/jcr.2015.0004.

7. Pergolizzi JV, Taylor R, Raffa RB. Intranasal ketorolac as part of a multimodal approach to postoperative pain. Pain Pract 2015; 15(4): 378-88. doi: 10.1111/ papr.12239.

8. Figueroa-Balderas L, Franco-Lopez F, Flores-Álvarez E, López-Rodríguez J, Vázquez-García J, BarbaValadez C. [Reduction of omalgia in laparoscopic cholecystectomy: clinical randomized trial ketorolac vs ketorolac and acetazolamide]. Cir Cir 2013; 81(5): 368-72. [In Spanish].

9. Schleiffarth JR, Bayon R, Chang KE, Van Daele DJ, Pagedar NA. Ketorolac after free tissue transfer a comparative effectiveness study. Ann Otol Rhinol Laryngol 2014; 123(6): 446-9.

10. Iorno V, Landi L, Di Pasquale R, Cicenia S, Moschini V. Comparison of intravenous ketorolac with or without paracetamol in postoperative pain control following ambulatory surgery. Curr Med Res Opin 2013; 29(12): 1685-90. doi: 10.1185/03007995.2013.835256

11. Beltrán-Montoya J, Herrerias-Canedo T, ArzolaPaniagua A, Vadillo-Ortega F, Dueñas-Garcia OF, Rico-Olvera H. A randomized, clinical trial of ketorolac tromethamine vs ketorolac trometamine plus complex B vitamins for cesarean delivery analgesia. Saudi J Anaesth 2012; 6(3): 207-12. doi: 10.4103/1658-354X.101209.

12. Buckley MM, Brogden RN. Ketorolac. A review of its pharmacodynamic and pharmacokinetic properties, and therapeutic potential. Drugs 1990; 39(1): 86-109. doi: 10.2165/00003495-199039010-00008.

13. Catapano MS. The analgesic efficacy of ketorolac for acute pain. J Emerg Med 1996; 14(1): 67-75. doi: 10.1016/0736-4679(95)02052-7.

14. Innes G, Croskerry P, Worthington J, Beveridge R, Jones D. Ketorolac versus acetaminophen-codeine in the emergency department treatment of acute low back pain. J Emerg Med 1998; 16(4): 549-56. doi: 10.1016/S0736-4679(98)00044-4/

15. Gillis JC, Brogden RN. Ketorolac. A reappraisal of its pharmacodynamic and pharmacokinetic properties and therapeutic use in pain management. Drugs 1997; 53(1): 139-88.

16. Hamel E. Current concepts of migraine pathophysiology. Can J Clin Pharmacol 1999; 6 Suppl
A: 9A-14A.

17. Williamson DJ, Hargreaves RJ. Neurogenic inflammation in the context of migraine. Microsc Res Tech 2001; 53(3): 167-78. doi: 10.1002/jemt.1081.

18. Kasmaei HD, Baratloo A, Nasiri Z, Soleymani M, Shirafkan A, Hamedi ZS. Report of nineteen cerebral vein thrombosis referrals to an emergency department: a case series and literature review. Arch Neurosci 2015; 2(2): e20552. doi: 10.5812/ archneurosci.20552.

19. Alimohammadi H, Baratloo A, Abdalvand A, Rouhipour A, Safari S. Effects of pain relief on arterial blood $\mathrm{o}_{2}$ saturation. Trauma Mon 2014; 19(1): e14034. doi: 10.5812/traumamon.14034.

20. Harden RN, Carter TD, Gilman CS, Gross AJ, Peters JR. Ketorolac in acute headache management. Headache 1991; 31(7): 463-64. doi:10.1111/j.1526-4610.1991. hed3107463.x

21. Meredith JT, Wait S, Brewer KL. A prospective double-blind study of nasal sumatriptan versus IV ketorolac in migraine. Am J Emerg Med 2003; 21(3): 173-5. doi: 10.1016/S0735-6757(02)42256-5.

22. Harden RN, Rogers D, Fink K, Gracely RH. Controlled trial of ketorolac in tension-type headache. Neurology 1998; 50(2): 507-9. doi: 10.1212/WNL.50.2.507.

23. Harden RN, Gracely RH, Carter T, Warner G. The placebo effect in acute headache management: ketorolac, meperidine, and saline in the emergency department. Headache 1996; 36(6): 352-6. doi: 10.1046/j.1526-4610.1996.3606352.x.

24. Wright JM, Price SD, Watson WA. NSAID use and efficacy in the emergency department: single doses of oral ibuprofen versus intramuscular ketorolac. Ann Pharmacother 1994; 28(3): 309-12.

25. Davis CP, Torre PR, Schafer NC, Dave B, Bass B. Ketorolac as a rapid and effective treatment of migraine headache: evaluations by patients. Am J Emerg Med 1993; 11(6): 573-5. doi: 10.1016/07356757(93)90003-T.

26. Friedman BW, Garber L, Yoon A, Solorzano C, Wollowitz A, Esses D, et al. Randomized trial of IV valproate vs metoclopramide vs ketorolac for acute migraine. Neurology 2014; 82(11): 976-83. doi: 10.1212/WNL.0000000000000223.

27. Friedman BW, Adewunmi V, Campbell C, Solorzano $\mathrm{C}$, Esses D, Bijur PE, et al. A randomized trial of intravenous ketorolac versus intravenous metoclopramide plus diphenhydramine for tensiontype and all nonmigraine, noncluster recurrent headaches. Ann Emerg Med 2013; 62(4): 311-18.e4. doi: 10.1016/j.annemergmed.2013.03.017. 